J. Korean Math. Soc. 53 (2016), No. 2, pp. 347-362

http://dx.doi.org/10.4134/JKMS.2016.53.2.347

\title{
ABSTRACT RANDOM LINEAR OPERATORS ON PROBABILISTIC UNITARY SPACES
}

\author{
Tran Xuan Quy, Dang Hung Thang, and Nguyen Thinh
}

\begin{abstract}
In this paper, we are concerned with abstract random linear operators on probabilistic unitary spaces which are a generalization of generalized random linear operators on a Hilbert space defined in [25]. The representation theorem for abstract random bounded linear operators and some results on the adjoint of abstract random linear operators are given.
\end{abstract}

\section{Introduction}

Let $(\Omega, \mathcal{F}, P)$ be a complete probability space and $X, Y$ be Banach spaces. A mapping $f: \Omega \times X \rightarrow Y$ is said to be a random operator (or a random mapping) defined on $X$ with values in $Y$ if for each $x \in X$, the mapping $\omega \mapsto f(\omega, x)$ is a $Y$-valued random variable. Equivalently, a random operator defined on $X$ with values in $Y$ is a mapping from $X$ into the space $L_{0}^{Y}(\Omega)$ of all $Y$-valued random variables. A random operator $f: X \rightarrow L_{0}^{Y}(\Omega)$ is said to be a random linear operator if $f$ is linear. The interest in random operators has been arouse not only for its own right as a random generalization of usual deterministic operators but also for their widespread applications in other areas. Research in theory of random operators has been carried out in many directions including random linear operators which provide a framework of stochastic integral, infinite random matrix (see e.g. [1], [2], [15]-[20], [23]$[26]$ ), random fixed points of random operators and random operator equations, (e.g [3]-[14], [18], [21], [22] and references therein). As an extension of random linear operators, generalized random linear operators on a separable Hilbert space were introduced and investigated in [25].

In this paper, generalized random linear operators on a separable Hilbert space are extended to abstract random linear operators on probabilistic unitary spaces. Section 2 presents the definitions and some properties of probabilistic

Received January 14, 2015; Revised September 22, 2015.

2010 Mathematics Subject Classification. 60H025, 47B80, 47H40, 60B11.

Key words and phrases. probabilistic linear space, probabilistic unitary space, probabilistic Hilbert space, abstract random linear operator, abstract random bounded linear operator, abstract random symmetric operator, abstract random self-adjoint operator, abstract random normal operator. 
unitary spaces and probabilistic Hilbert spaces in the connection with the notion of random normed modules introduced and investigated by some authors (see [4]-[8] and references therein). The main result of Section 2 is the theorem on the orthogonal decomposition of a probabilistic Hilbert space (Theorem 2.4). Next, in the Section 3 we deal with abstract random linear operators on probabilistic unitary spaces. It should be noted that generalized random linear operators on a Hilbert space defined in [25] can be regarded as a special case of abstract random linear operators. The main results of Section 3 is the representation theorem (Theorem 3.2) which states that an abstract random bounded linear operator from a probabilistic Hilbert space $\mathcal{H}$ into the space of complexvalued random variables is bounded if and only if it is represented as a random inner product on $\mathcal{H}$. Finally, in the Section 4 abstract random normal operators, abstract random symmetric operators and abstract random self-adjoint operators on a probabilistic Hilbert space $\mathcal{H}$ are introduced and studied. Applying the main results in Section 3, we obtain a sufficient condition for an abstract random symmetric operators can be extended to an abstract random self-adjoint operator (Theorem 4.2) and a claim that if $\Phi: \mathcal{D}(\Phi) \rightarrow \mathcal{H}$ is an abstract random self-adjoint operator and $\alpha$ is a complex number such that $\operatorname{Im}(\alpha) \neq 0$, then $\Phi_{\alpha}=\alpha I-\Phi: \mathcal{D}(\Phi) \rightarrow \mathcal{H}$ is bijective and $\left(\Phi_{\alpha}\right)^{-1}: \mathcal{H} \rightarrow \mathcal{H}$ is an abstract random normal operator (Theorem 4.3).

\section{Probabilistic unitary spaces and probabilistic Hilbert spaces}

Let $(\Omega, \mathcal{F}, P)$ be a complete probability space and $X$ be a complex separable Banach space. A mapping $u: \Omega \rightarrow X$ is said to be a $X$-valued random variable (r.v.) if $u$ is $(\mathcal{F}, \mathcal{B})$-measurable where $\mathcal{B}$ denotes the Borel $\sigma$-algebra of $X$. The space of all (equivalence classes of) $X$-valued r.v.'s is denoted by $L_{0}^{X}(\Omega)$. If $X$ is the field $\mathbb{C}$ of complex numbers, then $L_{0}^{\mathbb{C}}(\Omega)$ is denoted briefly by $L_{0}(\Omega)$. If $\xi_{1}, \xi_{2} \in L_{0}^{\mathbb{R}}(\Omega)$ we write $\xi_{1} \geqslant \xi_{2}$ if $\xi_{1}(\omega) \geqslant \xi_{2}(\omega)$ a.s. and $L_{0}^{+}(\Omega)=\{\xi \in$ $\left.L_{0}^{\mathbb{R}}(\Omega): \xi \geqslant 0\right\}$ denotes the set of all non-negative r.v's. The convergence in $L_{0}^{X}(\Omega)$ means the convergence in probability and we write p- $\lim _{n \rightarrow \infty} u_{n}=u$ if a sequence $\left(u_{n}\right) \in L_{0}^{X}(\Omega)$ converges to $u$ in $L_{0}^{X}(\Omega)$.

Definition (see [4]). An ordered pair $(S,\|\cdot\|)$ is called an random normed module over $\mathbb{C}$ if $S$ is a left module over the ring $L_{0}(\Omega)$ and $\|\cdot\|$ is a mapping from $S$ into $L_{0}^{+}(\Omega)$, called a random norm, such that the following conditions are satisfied

(1) $\|u\|=0$ if and only if $u=\theta$, where $\theta$ is the neutral element of $S$;

(2) $\|u+v\| \leqslant\|u\|+\|v\|$ for all $u, v \in S$;

(3) $\|\xi u\|=|\xi|\|u\|$ for each $\xi \in L_{0}(\Omega)$ and each $u \in S$.

Definition. A set $\mathcal{V}$ is said to be a probabilistic linear space if $\mathcal{V}$ is a module over the ring $L_{0}(\Omega)$. Namely 
(1) The operation of addition is defined in $\mathcal{V}$ and $\mathcal{V}$ is a commutative group with respect to this operation. The neutral element of $\mathcal{V}$ is denoted by $\theta$.

(2) The operation of multiplication by complex-valued random variables is defined in $\mathcal{V}$ and is commutative: For $u \in \mathcal{V}, \xi \in L_{0}(\Omega)$, then $\xi u \in$ $\mathcal{V}, u \xi \in \mathcal{V}, \xi u=u \xi$ and the following conditions are satisfied

$$
\xi(u+v)=\xi u+\xi v, \quad(\xi+\eta) u=\xi u+\eta u, \quad \text { and } \quad \xi(\eta u)=(\xi \eta) u .
$$

Example 1. Let $X$ be a complex separable Banach space and $V$ be a subset of $L_{0}^{X}(\Omega)$. For each $\xi \in L_{0}(\Omega), u, v \in L_{0}^{X}(\Omega)$ define $u+v$ by $(u+v)(\omega)=$ $u(\omega)+v(\omega)$ and $\xi u$ by $(\xi u)(\omega)=\xi(\omega) u(\omega)$. Denote by $\mathcal{V}$ the set of $X$-valued r.v.'s $u$ of the form

$$
u=\sum_{i=1}^{n} \xi_{i} v_{i}, \quad \xi \in L_{0}(\Omega), v_{i} \in V, n=1,2, \ldots
$$

It is easy to check that $\mathcal{V}$ is a probabilistic linear space.

Example 2. Let $X, Y$ be two complex separable Banach spaces and $\mathcal{V}(X, Y, \Omega)$ stands for the set of all random operators $\Phi: X \rightarrow L_{0}^{Y}(\Omega)$. For each $\Phi, \Psi \in$ $\mathcal{V}(X, Y, \Omega), \quad \xi \in L_{0}(\Omega)$ define $\Phi+\Psi$ by $(\Phi+\Psi) x=\Phi x+\Psi x$ and $\xi \Phi$ by $(\xi \Phi)(x)=\xi \Phi x$. It is easy to check that $\mathcal{V}(X, Y, \Omega)$ is a probabilistic linear space.

Definition. Let $\mathcal{V}$ be a probabilistic linear space. Assume that with every pair $u, v \in \mathcal{V}$ there is associated a complex-valued r.v. $h(u, v) \in L_{0}(\Omega)$, such that for every pair $u, v \in \mathcal{V}$ and for each $\xi \in L_{0}(\Omega)$ :

(1) $h\left(u_{1}+u_{2}, v\right)=h\left(u_{1}, v\right)+h\left(u_{2}, v\right)$;

(2) $h\left(u, v_{1}+v_{2}\right)=h\left(u, v_{1}\right)+h\left(u, v_{2}\right)$;

(3) $h(\xi u, v)=\xi h(u, v) ; \quad h(u, \xi v)=\bar{\xi} h(u, v)$;

(4) $h(u, v)=\overline{h(v, u)}$

(5) $h(u, u) \in L_{0}^{+}(\Omega)$ and $h(u, u)=0$ if and only if $u=\theta$.

$h(\cdot, \cdot)$ is called a random inner product on $\mathcal{V}$ and $\mathcal{V}$ is called a probabilistic unitary space with the random inner product $h(\cdot, \cdot)$.

Example 3. Let $H$ be a complex Hilbert space with the inner product $\langle\cdot, \cdot\rangle$ and $\mathcal{V} \subset L_{0}^{H}(\Omega)$ be a probabilistic linear space. Then $\mathcal{V}$ is a probabilistic unitary space with the random inner product $h(\cdot, \cdot)$ given by $h(u, v)(\omega)=\langle u(\omega), v(\omega)\rangle$.

From now on, a random inner product $h(\cdot, \cdot)$ on a probabilistic unitary space $\mathcal{V}$ will be denoted by $\langle\cdot, \cdot\rangle$.

Theorem 2.1. Let $\mathcal{V}$ be a probabilistic unitary space and $u, v \in \mathcal{V}$. Define $\|u\|=\sqrt{\langle u, u\rangle}$. Then

(1) $\|u\|=0$ if and only if $u=\theta$.

(2) $\|\xi u\|=|\xi|\|u\|$. 
(3) For $u, v \in \mathcal{V}, A, B \in \mathcal{F}, A \cap B=\emptyset$ we have

$$
\left\|1_{A} u+1_{B} v\right\|=1_{A}\|u\|+1_{B}\|v\| .
$$

(4)

$$
|\langle u, v\rangle| \leqslant\|u\|\|v\|
$$

$$
\|u+v\| \leqslant\|u\|+\|v\| .
$$

$$
\|u+v\|^{2}+\|u-v\|^{2}=2\left(\|u\|^{2}+\|v\|^{2}\right) .
$$

Proof. The proofs of (1)-(3) and (5)-(6) are clear. It suffices to prove (4). Let $\xi \in L_{0}(\Omega)$ be defined by

$$
\xi(\omega)= \begin{cases}\frac{\|u\|^{2}(\omega)}{\langle v, u\rangle(\omega)} & \text { if }\langle u, v\rangle(\omega) \neq 0 \\ 0 & \text { otherwise. }\end{cases}
$$

There is a set $D$ with $P(D)=1$ such that for each $\omega \in D$ one has $\langle u-\xi v, u-$ $\xi v\rangle(\omega) \geqslant 0$. Let $\omega \in D$. If $\langle u, v\rangle(\omega)=0$, then $|\langle u, v\rangle(\omega)| \leqslant\|u\|(\omega)\|v\|(\omega)$. If $\langle u, v\rangle(\omega) \neq 0$, then

$$
\begin{aligned}
\langle u-\xi v, u-\xi v\rangle(\omega) & =\|u\|^{2}(\omega)-\bar{\xi}(\omega)\langle u, v\rangle(\omega)-\xi(\omega)\langle v, u\rangle(\omega)+|\xi|^{2}(\omega)\|u\|^{2}(\omega) \\
& =-\|u\|^{2}(\omega)+\frac{\|u\|^{4}(\omega)}{|\langle u, v\rangle(\omega)|^{2}}\|v\|^{2}(\omega) \geqslant 0
\end{aligned}
$$

so $|\langle u, v\rangle(\omega)| \leqslant\|u\|(\omega)\|v\|(\omega)$. Hence, $|\langle u, v\rangle(\omega)| \leqslant\|u\|(\omega)\|v\|(\omega), \forall \omega \in D$, i.e., (2.1) holds.

From Theorem 2.1 we get:

Corollary 2.2. If $\mathcal{V}$ is a probabilistic unitary space, then it is a random normed module over $\mathbb{C}$.

Definition. Let $\mathcal{V}$ be a probabilistic unitary space.

(1) A sequence $\left(u_{n}\right) \subset \mathcal{V}$ is said to converge to $u \in \mathcal{V}$ if for every $\epsilon>0$ we have $\lim _{n \rightarrow \infty} P\left(\left\|u_{n}-u\right\|>\epsilon\right)=0$.

(2) A sequence $\left(u_{n}\right) \subset \mathcal{V}$ is said to be a Cauchy sequence if for each $\epsilon>0$ we have $\lim _{n, m \rightarrow \infty} P\left(\left\|u_{n}-u_{m}\right\|>\epsilon\right)=0$.

(3) $\mathcal{V}$ is said to be complete if every Cauchy sequence $\left(u_{n}\right) \subset \mathcal{V}$ is convergent.

(4) A probabilistic complete unitary space is called a probabilistic Hilbert space. From now on, a probabilistic Hilbert space is always denoted by $\mathcal{H}$.

Remark 1. Define a function $d$ on $\mathcal{V} \times \mathcal{V}$ by

$$
d(u, v)=\mathbb{E} \frac{\|u-v\|}{1+\|u-v\|} .
$$


It is easy to prove that $d$ is a metric on $\mathcal{V}$. $\lim _{n \rightarrow \infty} u_{n}=u$ if and only if $\lim _{n \rightarrow \infty} d\left(u_{n}, u\right)=0$ and $\left(u_{n}\right) \subset \mathcal{V}$ is a Cauchy sequence if and only if $\lim _{n, m \rightarrow \infty} d\left(u_{n}, u_{m}\right)=0$.

Lemma 2.3. Let $\mathcal{V}$ be a probabilistic unitary space with the random inner product $\langle\cdot, \cdot\rangle$. Then

(i) If $\left(u_{n}\right)$ and $\left(v_{n}\right)$ are two Cauchy sequences in $\mathcal{V}$, then $\left(\left\langle u_{n}, v_{n}\right\rangle\right)$ is a Cauchy sequence in $L_{0}(\Omega)$.

(ii) If $\lim _{n \rightarrow \infty} u_{n}=u$, $\lim v_{n \rightarrow \infty}=v$ in $\mathcal{V}$, then $\lim _{n \rightarrow \infty}\left\langle u_{n}, v_{n}\right\rangle=\langle u, v\rangle$ in $L_{0}(\Omega)$.

Proof. (i) Given $\epsilon>0$. Since $\lim _{n, m \rightarrow \infty} P\left(\left\|u_{n}-u_{m}\right\|>\epsilon\right)=0$ it follows that $\left(\left\|u_{n}\right\|\right)$ is a Cauchy in $L_{0}(\Omega)$. Hence the exists $\lim _{n \rightarrow \infty}\left\|u_{n}\right\|$ in $L_{0}(\Omega)$. Hence there is $c>0$ such that $P\left(\left\|u_{n}\right\|>c\right)<\epsilon, \forall n$. For each $t>0$ we have

$$
\begin{aligned}
P\left(\left|\left\langle u_{n}, v_{n}-v_{m}\right\rangle\right|>t\right) & \leqslant P\left(\left\|u_{n}\right\|\left\|v_{n}-v_{m}\right\|>t\right) \\
& \left.\leqslant P\left(\left\|u_{n}\right\|\left\|v_{n}-v_{m}\right\|>t\right),\left\|u_{n}\right\|<c\right)+P\left(\left\|u_{n}\right\|>c\right) \\
& \leqslant P\left(\left\|v_{n}-v_{m}\right\|>t / c\right)+\epsilon,
\end{aligned}
$$

so $\lim \sup _{n, m \rightarrow \infty} P\left(\left|\left\langle u_{n}, v_{n}-v_{m}\right\rangle\right|>t\right) \leqslant \epsilon$. By taking limit as $\epsilon \rightarrow 0$, we get

$$
\limsup _{n, m \rightarrow \infty} P\left(\left|\left\langle u_{n}, v_{n}-v_{m}\right\rangle\right|>t\right)=0 \text {. }
$$

Similarly

$$
\lim _{n, m \rightarrow \infty} P\left(\left|\left\langle u_{n}-u_{m}, v_{m}\right\rangle\right|>t\right)=0
$$

Since,

$$
\begin{aligned}
& P\left(\left|\left\langle u_{n}, v_{n}\right\rangle-\left\langle u_{m}, v_{m}\right\rangle\right|>t\right) \\
\leqslant & P\left(\left|\left\langle u_{n}, v_{n}-v_{m}\right\rangle\right|>t / 2\right)+P\left(\left|\left\langle u_{n}-u_{m}, v_{m}\right\rangle\right|>t / 2\right),
\end{aligned}
$$

we obtain that $\lim _{n, m \rightarrow \infty} P\left(\left|\left\langle u_{n}, v_{n}\right\rangle-\left\langle u_{m}, v_{m}\right\rangle\right|>t\right)=0$ as required.

The proof of (ii) is carried out by a similar argument.

Remark 2. If $\mathcal{V}$ is a probabilistic unitary space, then completing $\mathcal{V}$ with respect to the metric $(2.2)$ we get a probabilistic Hilbert space $\tilde{\mathcal{V}}$. The random inner product of two elements $\bar{u}, \bar{v} \in \tilde{\mathcal{V}}$ where $\bar{u}=\left(u_{n}\right), \bar{v}=\left(v_{n}\right)$ are two Cauchy sequences in $\mathcal{V}$ is given by

$$
\langle\bar{u}, \bar{v}\rangle_{\tilde{\mathcal{V}}}=\lim _{n \rightarrow \infty}\left\langle u_{n}, v_{n}\right\rangle \quad \text { in } L_{0}(\Omega) .
$$

Theorem 4.3 in [25] on the orthogonal decomposition of $L_{0}^{H}(\Omega)$ is extended to the following theorem on the orthogonal decomposition of a probabilistic Hilbert space.

Theorem 2.4. Let $\mathcal{H}$ be a probabilistic Hilbert space and $\mathcal{M} \subset \mathcal{H}$ be a closed probabilistic linear space. Then every element $u \in \mathcal{H}$ can be represented in a unique way as the sum

$$
u=v_{0}+u_{0}, \quad v_{0} \in \mathcal{M}, u_{0} \in \mathcal{M}^{\perp},
$$


where $\mathcal{M}^{\perp}=\{u \in \mathcal{H}:\langle u, v\rangle=0$ for all $v \in \mathcal{M}\}$.

The proof is carried out in almost the same as the proof of Theorem 4.3 in [25] with the notice that for $u, v \in \mathcal{H}$ and $D \in \mathcal{F}$ we have

$$
\mathbb{E} 1_{D}\|u\|^{2}+\mathbb{E} 1_{D^{c}}\|v\|^{2}=\mathbb{E}\left\|1_{D} u+1_{D^{c} v}\right\|^{2}
$$

by using Theorem 2.1 .

Hence if $\mathbb{E}\left\|1_{D} u+1_{D^{c}} v\right\|^{2} \geqslant \mathbb{E}\|v\|^{2}$, then

$$
\int_{D}\|u\|^{2} d P=\mathbb{E} 1_{D}\|u\|^{2} \geqslant \mathbb{E}\|v\|^{2}-E 1_{D^{c}}\|v\|^{2}=\int_{D}\|v\|^{2} d P .
$$

\section{Abstract random linear operators}

Let $\mathcal{V}, \mathcal{Y}$ be two probabilistic unitary spaces.

Definition. A mapping $\Phi: \mathcal{V} \rightarrow \mathcal{Y}$ is said to be an abstract random linear operator if for $u_{1}, u_{2} \in \mathcal{V}, \xi_{1}, \xi_{2} \in L_{0}(\Omega)$, then

$$
\Phi\left(\xi_{1} u_{1}+\xi_{2} u_{2}\right)=\xi_{1} \Phi\left(u_{1}\right)+\xi_{2} \Phi\left(u_{2}\right) .
$$

As usual, the domain $\mathcal{V}$ of $\Phi$ is denoted by $\mathcal{D}(\Phi)$.

Example 4. Let $A: H \rightarrow L_{0}^{H}(\Omega)$ be a random linear operator, where $H$ is a separable Hilbert space. Denote by $\mathcal{V}$ the subset of $L_{0}^{H}(\Omega)$ of the form

$$
u=\sum_{i=1}^{n} \xi_{i} x_{i},
$$

where $x_{i} \in H, \xi_{i} \in L_{0}(\Omega)$. Clearly, $\mathcal{V}$ is a probabilistic linear space containing $H$. By Example $3, \mathcal{V}$ is a probabilistic unitary space. Define a mapping $\Phi$ : $\mathcal{V} \rightarrow L_{0}^{H}(\Omega)$ by

$$
\Phi u=\sum_{i=1}^{n} \xi_{i} A x_{i}
$$

for $u$ is of the form given as in (3.1).

It is easy to verify that this definition is well-defined and $\Phi$ is an abstract random linear operator extending $A$.

Example 5. Let $A: H \rightarrow L_{0}^{H}(\Omega)$ be a random continuous linear operator, where $H$ is a Hilbert space with the basis $e=\left(e_{n}\right)_{n=1}^{\infty}$.

Denote by $\mathcal{V} \subset L_{0}^{H}(\Omega)$ the set of all $H$-valued r.v.'s $u$ for which the series

$$
\sum_{n=1}^{\infty}\left(u, e_{n}\right) A e_{n}
$$

converges in $L_{0}^{H}(\Omega)$. If $u \in \mathcal{V}$, then the sum (3.2) is denoted by $\Psi u$. 
It is easily shown that $\mathcal{V}$ is a probabilistic unitary space and the mapping $\Psi: \mathcal{V} \rightarrow L_{0}^{H}(\Omega)$ is an abstract random linear operator. Moreover since $A$ : $H \rightarrow L_{0}^{H}(\Omega)$ is continuous and

$$
x=\sum_{n=1}^{\infty}\left(x, e_{n}\right) e_{n}
$$

we get

$$
A x=\sum_{n=1}^{\infty}\left(x, e_{n}\right) A e_{n}
$$

which shows that $H \subset \mathcal{V}$ and $\Psi$ is an abstract random linear operator extending A.

Definition. Let $\Phi: \mathcal{V} \rightarrow \mathcal{Y}$ be an abstract random linear operator.

(1) $\Phi$ is said to be bounded if there exists a non-negative random variable $k \in L_{0}^{+}(\Omega)$ such that for each $u \in \mathcal{V}$ we have

$$
\|\Phi u\| \leqslant k\|u\| \text {. }
$$

(2) $\Phi$ is said to be continuous if for each sequence $\left(u_{n}\right) \subset \mathcal{V}$ such that $\lim _{n \rightarrow \infty} u_{n}=u \in \mathcal{V}$ we have $\lim _{n \rightarrow \infty} \Phi u_{n}=\Phi u$.

(3) $\Phi$ is said to be closed if for each sequence $\left(u_{n}\right) \subset \mathcal{V}$ such that $\lim _{n \rightarrow \infty} u_{n}$ $=u, \lim _{n \rightarrow \infty} \Phi u_{n}=g$ we have $u \in \mathcal{V}$ and $g=\Phi u$.

Theorem 3.1. Let $\Phi: \mathcal{V} \rightarrow \mathcal{Y}$ be an abstract random bounded linear operator. Then

(1) $\Phi$ is continuous.

(2) If $\left(u_{n}\right) \subset \mathcal{V}$ is a Cauchy sequence, then $\lim _{n \rightarrow \infty} \Phi u_{n}$ exists in $\mathcal{Y}$ provided that $\mathcal{Y}$ is complete.

Proof. (1) Let $u_{n} \in \mathcal{V}$ such that $\lim _{n \rightarrow \infty} u_{n}=u \in \mathcal{V}$. For each $\epsilon, r>0$ we have

$$
\begin{aligned}
P\left(\left\|\Phi u_{n}-\Phi u\right\|>\epsilon\right)= & P\left(\left\|\Phi u_{n}-\Phi u\right\|>\epsilon,\left\|u_{n}-u\right\| \leqslant r\right) \\
& +P\left(\left\|\Phi u_{n}-\Phi u\right\|>\epsilon,\left\|u_{n}-u\right\|>r\right) \\
\leqslant & P(k>\epsilon / r)+P\left(\left\|u_{n}-u\right\|>r\right) .
\end{aligned}
$$

Letting $n \rightarrow \infty$ then $r \rightarrow 0$ we get $\lim _{n \rightarrow \infty} P\left(\left\|\Phi u_{n}-\Phi u\right\|>\epsilon\right)=0$.

(2) Let $\left(u_{n}\right) \subset \mathcal{V}$ be a Cauchy sequence in $\mathcal{V}$. For each $\epsilon, r>0$ we have

$$
\begin{aligned}
P\left(\left\|\Phi u_{n}-\Phi u_{m}\right\|>\epsilon\right)= & P\left(\left\|\Phi u_{n}-\Phi u_{m}\right\|>\epsilon,\left\|u_{n}-u_{m}\right\| \leqslant r\right) \\
& +P\left(\left\|\Phi u_{n}-\Phi u_{m}\right\|>\epsilon,\left\|u_{n}-u_{m}\right\|>r\right) \\
\leqslant & P(k>\epsilon / r)+P\left(\left\|u_{n}-u_{m}\right\|>r\right) .
\end{aligned}
$$

Hence

$$
\limsup _{n, m \rightarrow \infty} P\left(\left\|\Phi u_{n}-\Phi u_{m}\right\|>\epsilon\right) \leqslant P(k>\epsilon / r) .
$$

Letting $r \rightarrow 0$ we obtain $\limsup _{n, m \rightarrow \infty} P\left(\left\|\Phi u_{n}-\Phi u_{m}\right\|>\epsilon\right)=0$, i.e., $\left(\Phi u_{n}\right)$ is a Cauchy sequence in $\mathcal{Y}$. Since $\mathcal{Y}$ is complete, there exists $\lim _{n \rightarrow \infty} \Phi u_{n}$. 
Now we prove the random version of the Riesz representation theorem for abstract random linear operator.

Theorem 3.2. Let $\mathcal{H}$ be a probabilistic Hilbert space and $\Gamma: \mathcal{H} \rightarrow L_{0}(\Omega)$ be an abstract random linear operator. Then $\Gamma$ is bounded if and only if there exists an element $w_{0} \in \mathcal{H}$ such that $\Gamma u=\left\langle u, w_{0}\right\rangle$ for every $u \in \mathcal{H}$.

Proof. Suppose that $\Gamma u=\left\langle u, w_{0}\right\rangle$ for every $u \in \mathcal{H}$. By the inequality (2.1) in Theorem 2.1 we have $|\Gamma u|=\left|\left\langle u, w_{0}\right\rangle\right| \leqslant\left\|w_{0}\right\|\|u\|$. Hence $\Gamma$ is bounded. Now we prove the converse.

If $\Gamma u=0$ for every $u \in \mathcal{H}$, then we take $w_{0}=0$. Otherwise, define $\mathcal{N}=$ $\{u: \Gamma u=0\} . \Gamma$ is bounded so it is continuous, thus $\mathcal{N}$ is a probabilistic closed linear space. The following lemma is crucial to the proof of Theorem 3.2.

Lemma 3.3. There exists $v_{0} \in \mathcal{N}^{\perp}$ such that $\mathcal{N}^{\perp}=\left\{u \in \mathcal{H}: u=\xi v_{0}, \xi \in\right.$ $\left.L_{0}(\Omega)\right\}$.

Proof of Lemma 3.3. For $u \in \mathcal{H}$, we denote $Z_{u}=\{\omega \in \Omega:\|u\|(\omega)=0\}$. If $A, B \in \mathcal{F}$, we write $A \subset B$ a.s if $1_{A} \leqslant 1_{B}$ and we write $A=B$ a.s if $1_{A}=1_{B}$. It is easy to see that if $A \subset B$ a.s., then $P(B \backslash A)=P(B)-P(A)$. On the set $\mathcal{N}^{\perp}$ we define a relation $\ll$ as follow: $u, v \in \mathcal{N}^{\perp}$

$$
u \ll v \text { if } Z_{v} \subset Z_{u} \text { a.s. and } 1_{Z_{u}^{c}} u=1_{Z_{u}^{c}} v .
$$

It is easy to check that the relation $\ll$ is reflexive, antisymmetric, and transitive, thus $\left(\mathcal{N}^{\perp}, \ll\right)$ is a partially ordered set.

Step 1. If $u \ll v$, then for each $\epsilon>0$

$$
P(\|u-v\|>\epsilon) \leqslant P\left(Z_{u}\right)-P\left(Z_{v}\right) .
$$

Indeed, put $A=Z_{v}, B=Z_{u}$. Then $A \subset B$ a.s. and $1_{B^{c}} u=1_{B^{c}} v$. Hence $1_{B^{c}}\|u-v\|=0,1_{A \cap B}\|u-v\| \leqslant 1_{A}\|u\|+1_{B}\|v\|=0$ which implies that

$$
\begin{aligned}
\|u-v\| & =1_{B^{c}}\|u-v\|+1_{A \cap B}\|u-v\|+1_{B \backslash A}\|u-v\| \\
& =1_{B \backslash A}\|u-v\| .
\end{aligned}
$$

Therefore, $P(\|u-v\|>\epsilon) \leqslant P(B \backslash A)=P(B)-P(A)=P\left(Z_{u}\right)-P\left(Z_{v}\right)$.

Step 2. There exists a maximal element $v_{0}$ of $\mathcal{N}^{\perp}$, i.e., if $u \in \mathcal{N}^{\perp}, v_{0} \ll u$, then $u=v_{0}$.

Proof of Step 2. By Zorn's Lemma it suffices to show that every totally ordered subset of $\left(\mathcal{N}^{\perp}, \ll\right)$ has an upper bound. Suppose $\mathcal{M}$ is a totally ordered subset of $\left(\mathcal{N}^{\perp}, \ll\right)$. Put $a=\inf \left\{P\left(Z_{u}\right), u \in \mathcal{M}\right\}$. Then there exists a sequence $u_{n} \in \mathcal{M}$ such that $u_{n} \ll u_{n+1}$ and $\lim _{n \rightarrow \infty} P\left(Z_{u_{n}}\right)=a$. Put $A_{n}=Z_{u_{n}}$. If $n>m$, then $u_{m} \ll u_{n}$. Hence by (3.4) $P\left(\left\|u_{m}-u_{n}\right\|>\epsilon\right) \leqslant P\left(A_{m}\right)-P\left(A_{n}\right)$ which implies that $\left(u_{n}\right)$ is a Cauchy sequence in $\mathcal{H}$. Since $\mathcal{N}^{\perp}$ is closed, there exists $u_{0} \in \mathcal{N}^{\perp}$ such that $\lim _{n \rightarrow \infty} u_{n}=u_{0}$. Put $A_{0}=Z_{u_{0}}$. If there is $m$ such that $u_{0} \ll u_{m}$, then $u_{0} \ll u_{n}, \forall n \geqslant m$. For each $n \geqslant m$ by (3.4) we have $P\left(\left\|u_{0}-u_{n}\right\|>\epsilon\right) \leqslant P\left(A_{0}\right)-P\left(A_{n}\right) \leqslant 0$, i.e., $P\left(\left\|u_{0}-u_{n}\right\|>\epsilon\right)=0$ for each $\epsilon>0$ which implies that $\left\|u_{0}-u_{n}\right\|=0 \rightarrow u_{0}=u_{n}$ for each $n \geqslant m$. 
Hence $u_{n} \ll u_{0} \forall n$. Now we show that $u \ll u_{0} \forall u \in \mathcal{M}$, i.e., $u_{0}$ is an upper bound of $\mathcal{M}$. Indeed, given $u \in \mathcal{M}$. If $u_{n} \ll u \forall n$, then $P\left(\left\|u-u_{n}\right\|>\epsilon\right) \leqslant$ $P\left(A_{n}\right)-P\left(Z_{u}\right)$. Hence $\lim \sup P\left(\left\|u-u_{n}\right\|>\epsilon\right) \leqslant a-P\left(Z_{u}\right) \leqslant 0$ implying that $\lim _{n \rightarrow \infty} P\left(\left\|u-u_{n}\right\|>\epsilon\right)=0$ so $u=u_{0}$. Otherwise, there is $u_{n}$ such that $u \ll u_{n} \ll u_{0}$ and we are done.

Step 3. For each $u \in \mathcal{N}^{\perp}$ we have

$$
Z_{v_{0}} \subset Z_{u} \text { a.s. }
$$

Indeed, let $u \in \mathcal{N}^{\perp}$. Put $w=1_{Z_{v_{0}}^{c}} v_{0}+1_{Z_{v_{0}} \backslash Z_{u}} u$. We have

$$
\|w\|=1_{Z_{v_{0}}}\left\|v_{0}\right\|+1_{Z_{v_{0}} \backslash Z_{u}}\|u\| \text { implies } Z_{w}=Z_{u} \cap Z_{v_{0}} \subset Z_{v_{0}} \text { a.s. }
$$

Moreover $1_{Z_{v_{0}}^{c}} v=1_{Z_{v_{0}}^{c}} w$. From this we deduce that $v_{0} \ll w$. Because $v_{0}$ is maximal so $v_{0}=w$ which implies $Z_{v_{0}}=Z_{w}$ a.s. Since $Z_{w}=Z_{u} \cap Z_{v_{0}} \subset Z_{u}$ a.s. we get (3.5) as claimed.

Step 4. At first we prove that for each $u \in \mathcal{N}^{\perp}$

$$
Z_{u}=Z_{\Gamma u} \quad \text { a.s. }
$$

Indeed, put $A=Z_{\Gamma u}, B=Z_{u}$ we have $1_{A}\|\Gamma u\|=0$, it follows that $1_{A} \Gamma u=0$ and hence $\Gamma\left(1_{A} u\right)=0$. Since $1_{A} u \in \mathcal{N}^{\perp}$ we get $1_{A} u=0$ which implies $1_{A}\|u\|=$ 0 and hence $A \subset B$ a.s.

Similarly, since $1_{B}\|u\|=0$ implies $1_{B} u=0$ and hence $\Gamma\left(1_{B} u\right)=0$ therefore $1_{B} \Gamma u=0$, implies $1_{B}|\Gamma u|=0$ so $B \subset A$ a.s. Therefore, $A=B$ a.s. as claimed.

Next, put

$$
\xi(\omega)= \begin{cases}\frac{\Gamma u(\omega)}{\Gamma v_{0}(\omega)} & \text { if } \Gamma v_{0}(\omega) \neq 0 \\ 0 & \text { if } \Gamma v_{0}(\omega)=0\end{cases}
$$

By (3.5) and (3.6) we get $Z_{\Gamma v_{0}}=Z_{v_{0}} \subset Z_{u}=Z_{\Gamma u}$ a.s. From (3.7) it follows that $\Gamma u=\xi \Gamma v_{0}=\Gamma\left(\xi v_{0}\right)$. Since $u, \xi v_{0} \in \mathcal{N}^{\perp}$ we conclude that $u=\xi v_{0}$. The proof of Lemma 3.3 is completed.

Put $A=\left\{\omega:\left\|v_{0}\right\|(\omega) \neq 0\right\}=Z_{v_{0}}^{c}$. Define $\lambda \in L_{0}(\Omega)$ by

$$
\lambda(\omega)= \begin{cases}\frac{\Gamma v_{0}(\omega)}{\left\|v_{0}\right\|^{2}(\omega)} & \text { if }\left\|v_{0}\right\|(\omega) \neq 0 \\ 0 & \text { if }\left\|v_{0}\right\|(\omega)=0\end{cases}
$$

we have $\lambda\left\|v_{0}\right\|^{2}=1_{A} \Gamma v_{0}$. Put $w_{0}=\bar{\lambda} v_{0} \in \mathcal{N}^{\perp}$. We shall show that for every $u \in \mathcal{H}$.

$$
\Gamma u=\left\langle u, w_{0}\right\rangle .
$$

Indeed, let $u \in \mathcal{H}$, by Theorem $2.4 u=u_{1}+u_{2}$ where $u_{1} \in \mathcal{N}, u_{2} \in \mathcal{N}^{\perp}$. Then

$$
\Gamma u=\Gamma u_{2},\left\langle u, w_{0}\right\rangle=\left\langle u_{2}, w_{0}\right\rangle .
$$


By Lemma 3.3 there exists $\xi \in L_{0}(\Omega)$ such that $u_{2}=\xi v_{0}$. Since $\left\langle u_{2}, w_{0}\right\rangle=$ $\xi\left\langle v_{0}, w_{0}\right\rangle$ we get

$$
\begin{aligned}
\left\|v_{0}\right\|^{2}\left\langle u_{2}, w_{0}\right\rangle & =\xi\left\langle v_{0},\left\|v_{0}\right\|^{2} w_{0}\right\rangle=\xi\left\langle v_{0},\left\|v_{0}\right\|^{2} \bar{\lambda} v_{0}\right\rangle \\
& =\xi \lambda\left\|v_{0}\right\|^{2}\left\langle v_{0}, v_{0}\right\rangle=\xi 1_{A} \Gamma v_{0}\left\langle v_{0}, v_{0}\right\rangle \\
& =1_{A} \Gamma\left(\xi v_{0}\right)\left\|v_{0}\right\|^{2}=1_{A} \Gamma u_{2}\left\|v_{0}\right\|^{2} .
\end{aligned}
$$

In addition we have

$$
Z_{v_{0}} \subset Z_{u} \quad \text { a.s. }|\Gamma u| \leqslant k\|u\|,\left|\left\langle u, w_{0}\right\rangle\right| \leqslant\|u\|\left\|w_{0}\right\| .
$$

Consequently combining with (3.10) there is a set $D$ with $P(D)=1$ such that if $\omega \in D$, then

$$
\begin{gathered}
\omega \in Z_{v_{0}} \quad \text { implies } \omega \in Z_{u}, \\
1_{A}(\omega) \Gamma u_{2}(\omega)\left\|v_{0}\right\|^{2}(\omega)=\left\|v_{0}\right\|^{2}(\omega)\left\langle u_{2}, w_{0}\right\rangle(\omega), \\
|\Gamma u(\omega)| \leqslant k(\omega)\|u\|(\omega), \\
\left|\left\langle u, w_{0}\right\rangle(\omega)\right| \leqslant\|u\|(\omega)\left\|w_{0}\right\|(\omega), \\
\Gamma u(\omega)=\Gamma u_{2}(\omega), \\
\left\langle u, w_{0}\right\rangle(\omega)=\left\langle u_{2}, w_{0}\right\rangle(\omega) .
\end{gathered}
$$

From this it follows that

- If $\omega \in Z_{v_{0}}^{c} \cap D$, then $\left\|v_{0}\right\|^{2}(\omega) \neq 0$ so $\Gamma u_{2}(\omega)=\left\langle u_{2}, w_{0}\right\rangle(\omega) \rightarrow \Gamma u(\omega)=$ $\left\langle u, w_{0}\right\rangle(\omega)$.

- If $\omega \in Z_{v_{0}} \cap D$ we have $\omega \in Z_{u} \rightarrow\|u\|(\omega)=0$. Hence $\Gamma u(\omega)=$ $\left\langle u, w_{0}\right\rangle(\omega)=0$.

Therefore $\Gamma u(\omega)=\left\langle u, w_{0}\right\rangle(\omega) \forall \omega \in D$, so the identity (3.9) is proved.

\section{The adjoint of abstract random linear operators}

Since in the sequence, we shall deal only with abstract random linear operators, we shall omit the adjective linear.

Definition. Given a probabilistic Hilbert space $\mathcal{H}$. Let $\Phi: \mathcal{D}(\Phi) \rightarrow \mathcal{H}$ be an abstract random operator with the dense domain $\mathcal{D}(\Phi) \subset \mathcal{H}$. Let $\mathcal{V}$ be the collection of $v \in \mathcal{H}$ for which there exists $g \in \mathcal{H}$ such that

$$
\langle\Phi u, v\rangle=\langle u, g\rangle
$$

for all $u \in \mathcal{D}(\Phi)$.

Because $\mathcal{D}(\Phi)$ is dense, such a $g$ is uniquely determined.

Putting $g=\Phi^{*} v$ we get a mapping $\Phi^{*}: \mathcal{V} \rightarrow \mathcal{H}$ and the domain $\mathcal{V}$ of $\Phi^{*}$ is denoted by $\mathcal{D}\left(\Phi^{*}\right)$. It is easy to check that $\mathcal{D}\left(\Phi^{*}\right)$ is a probabilistic linear space and $\Phi^{*}$ is an abstract random operator and is called the adjoint of $\Phi$. 
Definition. The adjoint $\Phi^{*}: \mathcal{D}\left(\Phi^{*}\right) \rightarrow \mathcal{H}$ is defined by the relation

$$
\langle\Phi u, v\rangle=\left\langle u, \Phi^{*} v\right\rangle
$$

for all $u \in \mathcal{D}(\Phi), v \in \mathcal{D}\left(\Phi^{*}\right)$.

Remark 3. The domains of two abstract random operators $\Phi$ and $\Psi$ introduced in Example 4 and Example 5 are dense in $L_{0}^{H}(\Omega)$.

Indeed it suffices to show that if a probabilistic unitary space $\mathcal{V} \subset L_{0}^{H}(\Omega)$ containing $H$ as a subset, then $\mathcal{V}$ is dense in $L_{0}^{H}(\Omega)$. Indeed, let $\mathcal{S}$ be the linear subspace of $L_{0}^{H}(\Omega)$ consisting simple r.v.'s. $u$ of the form

$$
u=\sum_{i=1}^{n} 1_{E_{i}} x_{i}, \quad x_{i} \in H, E_{i} \in \mathcal{F} .
$$

Since $H \subset \mathcal{V}$ it follows that $\mathcal{S} \subset \mathcal{V}$. Since $\mathcal{S}$ is dense in $L_{0}^{H}(\Omega)$ so is $\mathcal{V}$.

The following example shows that the domain of $\Phi^{*}$ need not be dense in $\mathcal{H}$.

Example 6. Let $\left(\xi_{n}\right)$ be a sequence of i.i.d real-valued r.v.'s such that $E \xi_{n}=$ $0, E\left|\xi_{n}\right|^{2}=1$. Let $\mathcal{V}$ be the set of $u \in L_{0}^{H}(\Omega)$ such that the series $\sum_{n=1}^{\infty}\left(u, e_{n}\right) \xi_{n}$ converges in probability. We claim that $\mathcal{V}$ is a probabilistic dense linear space. Clearly $\mathcal{V}$ is a probabilistic linear space. For each $x \in H$

$$
\sum_{n=1}^{\infty} E\left|\left(x, e_{n}\right) \xi_{n}\right|^{2}=\sum_{n=1}^{\infty}\left|<x, e_{n}>\right|^{2}=\|x\|^{2}<\infty
$$

which implies the series $\sum_{n=1}^{\infty}\left(x, e_{n}\right) \xi_{n}$ converges a.s. Hence $H \subset \mathcal{V}$. By the Remark 3, $\mathcal{V}$ is dense in $L_{0}^{H}(\Omega)$.

For $u \in \mathcal{V}$ put

$$
T u=\sum_{n=1}^{\infty}\left(u, e_{n}\right) \xi_{n} .
$$

Take $a \in H, a \neq 0$. Define a mapping $\Phi: \mathcal{V} \rightarrow L_{0}^{H}(\Omega)$ by $\Phi u=a T u$. It is easy to see that $\Phi$ is an abstract random operator.

Now we prove that $\mathcal{D}\left(\Phi^{*}\right)$ is not dense in $L_{0}^{H}(\Omega)$. Indeed $v \in \mathcal{D}\left(\Phi^{*}\right)$ if and only if there is $g \in L_{0}^{H}(\Omega)$ such that for all $u \in \mathcal{D}(\Phi)$

$$
\begin{gathered}
\langle\Phi u, v\rangle=\langle u, g\rangle \Leftrightarrow\langle a, v\rangle T u=\langle u, g\rangle \\
\rightarrow\langle a, v\rangle T e_{n}=\left\langle e_{n}, g\right\rangle \quad \forall n \\
\rightarrow\langle a, v\rangle \xi_{n}=\left\langle e_{n}, g\right\rangle \rightarrow|\langle a, v\rangle|^{2}\left|\xi_{n}\right|^{2}=\left|\left\langle e_{n}, g\right\rangle\right|^{2} \\
\rightarrow|\langle a, v\rangle|^{2} \sum_{n=1}^{\infty}\left|\xi_{n}\right|^{2}=\sum_{n=1}^{\infty}\left|\left\langle e_{n}, g\right\rangle\right|^{2}=\|g\|^{2} .
\end{gathered}
$$

It is easy to prove that $\sum_{n=1}^{\infty}\left|\xi_{n}\right|^{2}=+\infty$ a.s. Hence

$$
|\langle a, v\rangle|^{2}=0 \rightarrow\langle a, v\rangle=0 .
$$


Hence

$$
\mathcal{D}\left(\Phi^{*}\right)=\left\{v \in L_{0}^{H}(\Omega):\langle a, v\rangle=0\right\},
$$

i.e., $\mathcal{D}\left(\Phi^{*}\right)=L_{0}^{H_{1}}(\Omega)$ where $H_{1}=[a]^{\perp}$. Since $a \neq 0, \mathcal{D}\left(\Phi^{*}\right)$ is not dense in $L_{0}^{H}(\Omega)$ as claimed.

The following theorem is a generalization of the claim 4 of Theorem 2.5 in [25] to the case of probabilistic Hilbert spaces.

Theorem 4.1. If $\Phi: \mathcal{H} \rightarrow \mathcal{H}$ is an abstract random bounded operator, then $\mathcal{D}\left(\Phi^{*}\right)=\mathcal{H}$ and $\Phi^{*}: \mathcal{H} \rightarrow \mathcal{H}$ is also an abstract random bounded operator.

Proof. Fixed $v \in \mathcal{H}$. We shall show that, there exists $g \in \mathcal{H}$ such that for all $u \in \mathcal{H}$ we have

$$
\langle\Phi u, v\rangle=\langle u, g\rangle \text {. }
$$

Indeed let $\Gamma: \mathcal{H} \rightarrow L_{0}(\Omega)$ be defined by $\Gamma u=\langle\Phi u, v\rangle$. We claim that $\Gamma$ is an abstract random bounded operator. Indeed, since $\Phi$ is bounded, there is a non-negative r.v. $k$ such that $\|\Phi u\| \leqslant k\|u\|$. Hence

$$
|\Gamma u|=|\langle\Phi u, v\rangle| \leqslant\|\Phi u\|\|v\| \leqslant k\|v\|\|u\| \quad \forall u \in \mathcal{H}
$$

showing that $\Gamma$ is bounded. By Theorem 3.2 there is $g \in \mathcal{H}$ such that

$$
\Gamma u=\langle u, g\rangle, \text { i.e., }\langle\Phi u, v\rangle=\langle u, g\rangle \quad \forall u \in \mathcal{H} .
$$

Consequently $\mathcal{D}\left(\Phi^{*}\right)=\mathcal{H}$ and $\Phi^{*} v=g$. Moreover, we have

$$
|\langle u, g\rangle|=|\langle\Phi u, v\rangle| \leqslant k\|v\|\|u\| \quad \forall u, v \in \mathcal{H} .
$$

For $u=g$ we get $\|g\|^{2} \leqslant k\|v\|\|g\|$ implies $\|g\| \leqslant k\|v\|$ and hence $\left\|\Phi^{*} v\right\| \leqslant$ $k\|v\|$, which shows that $\Phi^{*}: \mathcal{H} \rightarrow \mathcal{H}$ is bounded.

Definition. Let $\Phi: \mathcal{D}(\Phi) \rightarrow \mathcal{H}$ be an abstract random operator with the dense domain $\mathcal{D}(\Phi) \subset \mathcal{H}$.

(1) $\Phi$ is said to be symmetric if $\langle\Phi u, v\rangle=\langle u, \Phi v\rangle \forall u, v \in \mathcal{D}(\Phi)$, i.e., $\mathcal{D}(\Phi) \subset$ $\mathcal{D}\left(\Phi^{*}\right)$ and $\Phi u=\Phi^{*} u \forall u \in \mathcal{D}(\Phi)$.

(2) $\Phi$ is said to be self-adjoint if $\Phi=\Phi^{*}$, i.e., $\mathcal{D}(\Phi)=\mathcal{D}\left(\Phi^{*}\right)$ and $\Phi u=$ $\Phi^{*} u \forall u \in \mathcal{D}(\Phi)$.

(3) $\Phi$ is said to be normal if $\mathcal{D}(\Phi)=\mathcal{H}, \Phi$ is bounded and $\Phi \Phi^{*}=\Phi^{*} \Phi$.

Notice that if $\Phi$ is symmetric, then $\langle\Phi u, u\rangle$ is a real-valued r.v. since $\langle\Phi u, u\rangle=$ $\langle u, \Phi u\rangle=\overline{\langle\Phi u, u\rangle}$.

Definition. Let $\Phi: \mathcal{D}(\Phi) \rightarrow \mathcal{H}$ be an abstract random symmetric operator.

(1) $\Phi$ is said to be bounded from below if there is a real-valued r.v. $m$ such that for each $u \in \mathcal{D}(\Phi)\langle\Phi u, u\rangle \geqslant m\|u\|^{2}$.

(2) $\Phi$ is said to be bounded from above if there is a real-valued r.v. $M$ such that for each $u \in \mathcal{D}(\Phi)\langle\Phi u, u\rangle \leqslant M\|u\|^{2}$.

(3) $\Phi$ is said to be semi-bounded if $\Phi$ is bounded from below or from above. 
Theorem 4.2. Given an abstract random semi-bounded symmetric operator $\Phi: \mathcal{D}(\Phi) \rightarrow \mathcal{H}$, there exists an abstract random self-adjoint operator $\tilde{\Phi}$ extending $\Phi$.

Proof. Since $\Phi$ is bounded from above if and only if $-\Phi$ is bounded from below, it suffices to assume that $\Phi$ is bounded from below. Without loss of generality we may suppose that $m=1$. Indeed, put $\Psi=\Phi-a I$ where $a=m-1$ then $\Psi$ is a probabilistic symmetric operator satisfying $\mathcal{D}(\Phi)=\mathcal{D}(\Psi), \mathcal{D}\left(\Phi^{*}\right)=\mathcal{D}\left(\Psi^{*}\right)$ and

$$
\langle\Psi u, u\rangle=\langle\Phi u, u\rangle-a\langle u, u\rangle \geqslant m\|u\|^{2}-m\left\|u^{2}\right\|+\|u\|^{2}=\|u\|^{2} .
$$

Let $\mathcal{M}=\mathcal{D}(\Phi)$. Define a random inner $\langle u, v\rangle_{\mathcal{M}}$ on $\mathcal{M}$ by $\langle u, v\rangle_{\mathcal{M}}=\langle\Phi u, v\rangle$. By assumption we have $\|u\| \leqslant\|u\|_{\mathcal{M}}$. Completing $\mathcal{M}$ with respect to the metric (2.2) induced by the random norm $\|u\|_{\mathcal{M}}$ we get a probabilistic Hilbert space $\tilde{\mathcal{M}}$. Because $\|u\|_{\mathcal{M}} \geqslant\|u\|$, each Cauchy sequence $\left(u_{n}\right)$ in $\mathcal{M}$ converges in $\mathcal{H}$ thus we can identify $\tilde{\mathcal{M}}$ with some subset of sequences converging in $\mathcal{H}$, i.e., $\tilde{\mathcal{M}}$ is the subset of $\mathcal{H}$.

Put $\mathcal{N}=\mathcal{D}\left(\Phi^{*}\right) \cap \tilde{\mathcal{M}}$. Since $\Phi$ is symmetric, we have $\mathcal{M} \subset \mathcal{D}\left(\Phi^{*}\right)$ so $\mathcal{M} \subset \mathcal{N} \subset \mathcal{D}\left(\Phi^{*}\right)$. Let $\tilde{\Phi}: \mathcal{N} \rightarrow \mathcal{H}$ be the restriction of $\Phi^{*}$ on $\mathcal{N}$. We claim that $\tilde{\Phi}$ is self-adjoint. Indeed,

- $\tilde{\Phi}$ is symmetric: Let $u, v \in \mathcal{N}$. Since $\mathcal{N} \subset \tilde{\mathcal{M}}$ there sequences $\left(u_{n}\right),\left(v_{n}\right)$ of elements of $\mathcal{M}$ such that $\lim _{n \rightarrow \infty} u_{n}=u$, $\lim _{n \rightarrow \infty} v_{n}=v$ in $\tilde{\mathcal{M}}$. We have

$$
\lim _{m \rightarrow \infty} \lim _{n \rightarrow \infty}\left\langle\Phi u_{n}, v_{m}\right\rangle=\lim _{m \rightarrow \infty} \lim _{n \rightarrow \infty}\left\langle u_{n}, v_{m}\right\rangle_{\mathcal{M}}=\lim _{m \rightarrow \infty}\left\langle u, v_{m}\right\rangle_{\mathcal{M}}=\langle u, v\rangle_{\mathcal{M}}
$$

Similarly $\lim _{n \rightarrow \infty} \lim _{m \rightarrow \infty}\left\langle\Phi u_{n}, v_{m}\right\rangle=\lim _{n \rightarrow \infty}\left\langle u_{n}, v\right\rangle_{\mathcal{M}}=\langle u, v\rangle_{\mathcal{M}}$. Hence

$$
\lim _{m \rightarrow \infty} \lim _{n \rightarrow \infty}\left\langle\Phi u_{n}, v_{m}\right\rangle=\lim _{n \rightarrow \infty} \lim _{m \rightarrow \infty}\left\langle\Phi u_{n}, v_{m}\right\rangle \text {. }
$$

On the other hand, since

$$
\lim _{n \rightarrow \infty} u_{n}=u, \lim _{n \rightarrow \infty} v_{n}=v \text { in } \mathcal{H},
$$

we also have

$$
\begin{aligned}
\lim _{m \rightarrow \infty} \lim _{n \rightarrow \infty}\left\langle\Phi u_{n}, v_{m}\right\rangle & =\lim _{m \rightarrow \infty} \lim _{n \rightarrow \infty}\left\langle u_{n}, \Phi v_{m}\right\rangle=\lim _{m \rightarrow \infty}\left\langle u, \Phi v_{m}\right\rangle \\
& =\lim _{m \rightarrow \infty}\left\langle\Phi^{*} u, v_{m}\right\rangle=\langle\tilde{\Phi} u, v\rangle .
\end{aligned}
$$

Similarly,

$$
\lim _{n \rightarrow \infty} \lim _{m \rightarrow \infty}\left\langle\Phi u_{n}, v_{m}\right\rangle=\lim _{n \rightarrow \infty}\left\langle u_{n}, \Phi^{*} v\right\rangle=\lim _{n \rightarrow \infty}\left\langle u, \Phi^{*} v\right\rangle=\langle u, \tilde{\Phi} v\rangle .
$$

Hence $\langle\tilde{\Phi} u, v\rangle=\langle u, \tilde{\Phi} v\rangle$ which proves that $\tilde{\Phi}$ is symmetric.

- The range $\mathcal{R}(\tilde{\Phi})$ is the whole space $\mathcal{H}$ : Let $v$ be any element of $\mathcal{H}$. Define $\Gamma: \tilde{\mathcal{M}} \rightarrow L_{0}$ by $\Gamma u=\langle u, v\rangle$. We have $|\Gamma u| \leqslant\|v\|\|u\| \leqslant\|v\|\|u\|_{\mathcal{M}}$ for all $u \in \tilde{\mathcal{M}}$. By Theorem 3.2 there is $v^{*} \in \tilde{\mathcal{M}}$ such that $\Gamma u=\left\langle u, v^{*}\right\rangle_{\mathcal{M}} \forall u \in \tilde{\mathcal{M}}$. Hence, for each $u \in \mathcal{M}\left\langle\Phi u, v^{*}\right\rangle=\left\langle u, v^{*}\right\rangle_{\mathcal{M}}=\langle u, v\rangle$. Hence $v^{*} \in \tilde{\mathcal{M}} \cap \mathcal{D}\left(\Phi^{*}\right)=\mathcal{N}$ and $v=\Phi^{*} v^{*}=\tilde{\Phi} v^{*}$. 
- $\tilde{\Phi}$ is injective: Indeed, suppose that $\tilde{\Phi} u=\theta$. Since $\mathcal{R}(\tilde{\Phi})=\mathcal{H}$ there is $v \in$ $\mathcal{D}(\tilde{\Phi})$ such that $u=\tilde{\Phi} v$. Then $\langle u, u\rangle=\langle u, \tilde{\Phi} v\rangle=\langle\tilde{\Phi} u, v\rangle=0$ implies $u=\theta$.

- $\Phi$ is self-adjoint: Since $\tilde{\Phi}$ is injective and $\mathcal{R}(\tilde{\Phi})=\mathcal{H}$ there exists $\Psi=$ $\tilde{\Phi}^{-1}: \mathcal{H} \rightarrow \mathcal{H}$. Since $\tilde{\Phi}$ is symmetric, $\Psi$ is also symmetric. Since $\mathcal{D}(\Psi)=\mathcal{H}$ it follows that $\Psi=\Psi^{*}$. By the standard argument similar to that in the case of deterministic linear operators we get $\Psi^{*}=\left(\tilde{\Phi}^{*}\right)^{-1}$. Hence $(\tilde{\Phi})^{-1}=$ $\left(\tilde{\Phi}^{*}\right)^{-1}$ implies $\tilde{\Phi}=\tilde{\Phi}^{*}$ as claimed.

The following theorem is a generalization of Theorem 4.2 in [25] to the case of probabilistic Hilbert spaces.

Theorem 4.3. Let $\Phi: \mathcal{D}(\Phi) \rightarrow \mathcal{H}$ be an abstract random self-adjoint operator and $\alpha$ is a complex number such that $\operatorname{Im}(\alpha) \neq 0$. Put $\Phi_{\alpha}=\alpha I-\Phi$. Then $\Phi_{\alpha}: \mathcal{D}(\Phi) \rightarrow \mathcal{H}$ is bijective and the inverse $\left(\Phi_{\alpha}\right)^{-1}: \mathcal{H} \rightarrow \mathcal{H}$ is an abstract random normal operator.

Proof. At first remark that:

Lemma 4.4. (1) Let $\Phi: \mathcal{D}(\Phi) \rightarrow \mathcal{H}$ be an abstract random self-adjoint operator. Then $\Phi$ is closed.

(2) Let $\Phi: \mathcal{D}(\Phi) \rightarrow \mathcal{H}$ be an abstract random closed bounded operator. Then the set $\mathcal{D}(\Phi)$ is closed.

Proof. (1) Let $\left(v_{n}\right) \subset \mathcal{D}(\Phi), \lim _{n \rightarrow \infty} v_{n}=v$ and $\lim _{n \rightarrow \infty} \Phi v_{n}=g$. Then for each $u \in \mathcal{D}(\Phi)$

$$
\langle u, g\rangle=\lim _{n \rightarrow \infty}\left\langle u, \Phi v_{n}\right\rangle=\lim _{n \rightarrow \infty}\left\langle\Phi u, v_{n}\right\rangle=\langle\Phi u, v\rangle .
$$

This show that $v \in \mathcal{D}\left(\Phi^{*}\right)$ and $\Phi^{*} v=g$. Since $\Phi=\Phi^{*}$ we conclude that $v \in \mathcal{D}(\Phi)$ and $\Phi v=g$.

(2) Assume that $\left(u_{n}\right) \subset \mathcal{D}(\Phi)$ such that $\lim _{n \rightarrow \infty} u_{n}=u$. By Theorem 3.1 there exists $\lim _{n \rightarrow \infty} \Phi u_{n}=g$. Since $\Phi$ is closed we conclude that $u \in \mathcal{D}(\Phi)$.

The proof of Theorem 4.3 is based on Lemma 4.4, Theorem 2.4 and the argument similar to that in the proof of Theorem 4.2 in [25].

Acknowledgement. We would like to express our gratitude to the referee for his detailed comments which help to produce a more compact exposition and to eliminate several confusing expressions from the first manuscript. This research is funded by Vietnam National Foundation for Science and Technology Development (NAFOSTED)under grand number 101.03-2013.

\section{References}

[1] A. A. Dorogovstev, On application of a Gaussian random operator to random elements, Theory Probab. Appl. 30 (1986), no. 4, 812-814.

[2] H. W. Engl, M. Z. Nashed, and M. Zuhair, Generalized inverses of random linear operators in Banach spaces, J. Math. Anal. Appl. 83 (1981), no. 2, 582-610.

[3] H. W. Engl and W. Romisch, Approximate solutions of nonlinear random operator equations: Convergence in distribution, Pacific J. Math. 120 (1985), no. 1, 55-77. 
[4] T. Guo, Module homomorphisms on random normed modules, Northeast. Math. J. 12 (1996), no. 1, 102-114.

[5] _ Relations between some basic results derived from two kinds of topologies for a random locally convex module, J. Funct. Anal. 258 (2010), no. 9, 3024-3047.

[6] T. Guo and G. Shi, The algebraic structure of finitely generated $L^{0}(\mathcal{F}, K)$-modules and the Helly theorem in random normed modules, J. Math. Anal. Appl. 381 (2011), no. 2, $833-842$.

[7] T. Guo and Y. Yang, Ekelands variational principle for an $\bar{L}^{0}$-valued function on a complete random metric space, J. Math. Anal. Appl. 389 (2012), no. 1, 1-14.

[8] Wu. Mingzhu, The Bishop-Phelps theorem in complete random normed modules endows with the $(\varepsilon, \lambda)$-topology, J. Math. Anal. Appl. 391 (2012), 648-652.

[9] M. Z. Nashed and H. W. Engl, Random generalized inverses and approximate solution of random equations, In: A. T. Bharucha-Reid (Ed.) Approximate Solution of random equations, pp. 149-210, Elsevier /North-Holland, New York-Amsterdam, 1979.

[10] H. Olga and P. Endre, Fixed Point Theory in Probabilistic Metric Spaces, Kluwer Academic Publishers, 2001.

[11] B. Schweizer and A. Sklar, Probabilistic Metric Spaces, Elsevier, New York, 1983.

[12] N. Shahzad, Random fixed points of $K$-set and pseudo-contractive random maps, Nonlinear Anal. 57 (2004), no. 2, 173-181.

[13] _ Random fixed point results for continuous pseudo-contractive random maps, Indian J. Math. 50 (2008), no. 2, 331-337.

[14] N. Shahzad and N. Hussain, Deterministic and random coincidence point results for f-nonexpansive maps, J. Math. Anal. Appl. 323 (2006), no. 2, 1038-1046.

[15] A. V. Skorokhod, Random Linear Operators, Reidel Publishing Company, Dordrecht, 1984.

[16] D. H. Thang, Random Operator in Banach spaces, Probab. Math. Statist. 8 (1987), $155-157$.

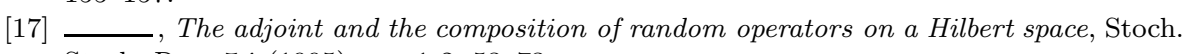
Stoch. Rep. 54 (1995), no. 1-2, 53-73.

[18] _ Random mappings on infinite dimensional spaces, Stoch. Stoch. Rep. 64 (1998), no. $1-2,51-73$.

[19] Series and spectral representations of random stable mappings, Stoch. Stoch. Rep. 64 (1998), no. 1-2, 33-49.

[20] - Transforming random operators into random bounded operators, Random Oper. Stoch. Equ. 16 (2008), no. 3, 293-302.

[21] D. H. Thang and P. T. Anh, Random fixed points of completely random operators, Random Oper. Stoch. Equ. 21 (2013), no. 1, 1-20.

[22] D. H. Thang and T. N. Anh, On random equations and applications to random fixed point theorems, Random Oper. Stoch. Equ. 18 (2010), no. 3, 199-212.

[23] D. H. Thang and T. M. Cuong, Some procedures for extending random operators, Random Oper. Stoch. Equ. 17 (2009), no. 4, 359-380.

[24] D. H. Thang and Ng. Thinh, Random bounded operators and their extension, Kyushu J. Math. 58 (2004), no. 2, 257-276.

[25] - Generalized random linear operators on a Hilbert space, Stochastics 85 (2013), no. 6, 1040-1059.

[26] D. H. Thang, Ng. Thinh, and Tr. X. Quy, Generalized random spectral measures, J. Theoret. Probab. 27 (2014), no. 2, 576-600. 
Tran XUAN QUY

Department of Mathematics

College of SCIEnCE

Thai Nguyen University

Thai Nguyen, Vietnam

E-mail address: quytx@tnu.edu.vn

DAng Hung Thang

Department of Mathematics

HANOI UNIVERSITY OF SCIENCES

HANOI, VieTnAM

E-mail address: thangdh@vnu.edu.vn

Nguyen Thinh

Department of Mathematics

Hanoi University of ScIEnces

HANOI, VIETNAM

E-mail address: nguyenthinh@vnu.edu.vn 